\title{
EDITORIAL
}

\section{Call for a randomized controlled trial: early glycemic control in the development of retinopathy of prematurity}

Journal of Perinatology (2014) 34, 415-416; doi:10.1038/jp.2014.50

In this edition of the Journal of Perinatology, Mohsen et al. ${ }^{1}$ present the first prospective study on the effect of elevated glucose on the development of retinopathy of prematurity (ROP). While the correlation between elevated glucose and ROP has been well delineated by several retrospective studies, Mohsen et al. ${ }^{1}$ are the first to conduct a prospective study and are able to confirm that elevated average neonatal glucose concentrations in the first week of life are independently associated with the development of ROP.

Concerns about the impact of exogenous insulin as an independent risk factor for ROP have confounded the discussions on the correlation of hyperglycemia with ROP. Mohsen et al.'s ${ }^{1}$ elegant avoidance of insulin use allowed for the conclusion that the effects of elevated glucose are linked to ROP and not exogenous insulin infusions, which other studies had not been able to exclude.

Another clever design decision was the use of only regularly scheduled glucose tests in the analysis. This approach nullified the known effect of abnormal glucose results leading to more testing and more abnormal results. If all glucose results had been used, episodes of hyper- and hypoglycemia would have received exaggerated weight due to the more frequent testing around the events with the result of a distorted or skewed picture. As only the regularly scheduled (every $8 \mathrm{~h}$ for the duration of a week) glucose laboratory tests were used, there was no need to apply the computationally expensive area-underthe-curve methodology (time-weighted) used by Chavez-Valdez et $a l^{2}$

What are the determining factors of today's understanding that ROP is correlated to early glycemic state? In part, the answer is serendipity. In 1998, attending a dinner with a lecture on adult diabetes as a guest of an invited family medicine physician, the talk had little relevance for me as a neonatology fellow. At one point, the lecturer discussed the complications of diabetes including 'neo-proliferative eye disease'. Having heard of 'neoproliferative eye disease' before, but in conjunction with premature infants' ROP, the remainder of the evening allowed the formulation of a novel question: Could the development of ROP also have a connection to neonatal glucose levels?

A literature search in the next few months discovered indirect evidence for a connection between elevated glucose and ROP. Events in the early life of premature infants that are usually associated with elevated glucose levels such as candida sepsis or the use of steroids had already been connected to the development of ROP. Absence of hypoglycemia also had been identified as a risk factor for ROP. Obviously, at that time the association of an important player in metabolism-oxygen-had been long established and oxygen was already administered in a controlled manner to reduce ROP risk.

A quick 'sanity check' that involved the review of the charts of five infants with severe ROP suggested evidence of a link to elevated glucose. However, distracted by a fellowship in Clinical Informatics, the plan to explore the role of glucose in ROP went into the 'ideas to pursue one day' file until in 2001, a pediatric resident requested a project for her research rotation. Dr Garg's determination and extraordinary work led in 2003 to the publication of the first retrospective study-also in the Journal of Perinatology - correlating glucose levels in the first month of life to the development of ROP. ${ }^{3}$

From the first serendipitous notion that glucose may be linked to ROP and from the first published study, 16 years and 11 years have passed respectively until today's publication of the first prospective study. Distractions may be to blame for the first 5-year delay, but the evidence has now been published and available for more than 10 years. ${ }^{3}$ Unfortunately, today we are not much closer to putting this knowledge into use in the prevention of ROP. Awareness of the correlation between glucose and ROP has not made it in the minds of the public. The current entry for ROP on Wikipedia does not include glucose as a risk factor. ${ }^{4}$

To date, the correlation between ROP and glucose has also not achieved the deserved attention by neonatologists. Glucose in newborn intensive care units is currently not managed with the intention to prevent ROP. No clinical trials have been conducted to evaluate approaches, safety and outcomes on ROP of tighter glucose control in the newborn intensive care unit in the first month of life, despite the evidence that glycemic protocols can be implemented safely in adult intensive care units ${ }^{5}$ and in neonatal intensive care units. ${ }^{6}$

In the 1950s, ROP was a disease considered extinct until new technologies and treatments permitted the survival of ever smaller and more premature infants. With the improved survival came an increase in the incidence ROP. In the history of this disease, the enormous time delay between recognition of disease correlation and clinical trials translates into tremendous opportunity loss. Time is of the essence! We need clinical trials on the management of acute hyperglycemia and exposure to prolonged elevated glucose levels in neonates with ROP as an outcome measure. It is time to develop and test new measures and approaches that will allow neonatologists to reduce the risk of blindness and loss of vision in their tiny patients.

\section{CONFLICT OF INTEREST}

Dr Lehmann serves on the board of the International Medical informatics Association and directs the Child Health Informatics Center at the American Academy of Pediatrics. He is the editor of both the journal Applied Clinical Informatics and the textbook Pediatric Informatics.

\author{
CU Lehmann ${ }^{1,2}$ \\ ${ }^{1}$ Department of Pediatrics, Vanderbilt University, Nashville, TN, USA \\ and \\ ${ }^{2}$ Department of Biomedical Informatics, Vanderbilt University, \\ Nashville, TN, USA \\ E-mail: Christoph.U.Lehmann@Vanderbilt.edu
}

\section{REFERENCES}

1 Mohsen L, Abou-Alam M, El-Dib M, Labib M, Elsada M, Mohsen HA et al. A prospective study on hyperglycemia and retinopathy of prematurity. J Perinatol 2014; 34(6) 453-457. 
2 Chavez-Valdez R, McGowan J, Cannon E, Lehmann CU. Contribution of early glycemic status in the development of severe retinopathy of prematurity in a cohort of ELBW infants. J Perinatol 2011; 31(12) 749-756.

3 Garg R, Agthe AG, Donohue PK, Lehmann CU. Hyperglycemia and retinopathy of prematurity in very low birth weight infants. J Perinatol 2003; 23(3) 186-194.

4 Wikipedia. Retinopathy of prematurity. Available online at http://en.wikipedia.org/ wiki/Retinopathy_of_prematurity. Last accessed 2/17/2014.
5 Martinez EA, Chavez-Valdez R, Holt NF, Grogan KL, Khalifeh KW, Slater T et al. Successful implementation of a perioperative glycemic control protocol in cardiac surgery: barrier analysis and intervention using lean six sigma. Anesthesiol Res Pract 2011; 2011: 565069.

6 Hebson CL, Chanani NK, Rigby MR, Wolf MJ, Deshpande SR, Montegna LM et al Safe and effective use of a glycemic control protocol for neonates in a cardiac ICU. Pediatr Crit Care Med 2013; 14(3) 284-289. 